\title{
PRESCRIBING PATTERN OF PSYCHOTROPIC AGENTS IN RURAL TERTIARY CARE TEACHING HOSPITAL
}

\author{
RAJESH VENKATARAMAN*, MADAN RAYAMAJHI, SHAHINUR ISLAM, NANJUNDA N
}

Department of Clinical Pharmacy, Sri Adichunchanagiri College of Pharmacy, B.G Nagara, Karnataka, India. Email: rajeshvenky_research@hotmail.com

Received: 03 November 2017, Revised and Accepted: 30 November 2017

\begin{abstract}
Objectives: The objectives of the study were to study the prescribing pattern on psychotropic agents at psychiatric department at rural tertiary care teaching hospital, B.G. Nagara.

Methods: A prospective and observational study was conducted among psychiatric department (either sex) after considering inclusion and exclusion criteria for the period of 6 months and a total of 300 prescriptions were analyzed.

Results: A total of 300 psychiatric patients were enrolled, male 170 (56.67\%) patients predominated over females 130 (43.33\%) patients. Majority of participants were under age group of 31-40 years (28.6\%), illiterate (75\%), unemployed (52\%), and married (84\%). Prevalence of mood disorder 109 (36.33\%) was high followed by substance used and addictive disorder 53 (17.67\%), schizophrenia 46 (15.33\%), and psychosomatic medicine 22 (7.33\%). Antidepressant (28.59\%) was the commonly prescribed group followed by benzodiazepines (14.81\%), anticholinergic (7.18\%), and anticonvulsants (7.18\%). Tricyclic antidepressants drugs amitriptyline (53.44\%) was most frequently prescribed an antidepressant. An average number of drugs per prescription was 2.32 out of which $16.91 \%$ of drugs were prescribed by their generic name.
\end{abstract}

Conclusion: Selective serotonin reuptake inhibitors (SSRIs) were the most common group, and escitalopram was the most common medication used among the SSRIs. Majority of the drugs were prescribed from essential drug list. This study advocated an overall rational utilization of psychotropic drugs with fewer deviations due to the socioeconomic status of patients and prescription practices of healthcare providers and need to improve prescribing habits to ensure rational use.

Keywords: Psychotropic agents, Prescription patterns, WHO guidelines.

(C) 2018 The Authors. Published by Innovare Academic Sciences Pvt Ltd. This is an open access article under the CC BY license (http://creativecommons. org/licenses/by/4. 0/) DOI: http://dx.doi.org/10.22159/ajpcr.2018.v11i3.23063

\section{INTRODUCTION}

An integral part of the primary healthcare system is the level of care responsible for providing basic healthcare services and taking care of public in the rural areas, without availability of necessary medicines modern healthcare system is impossible [1]. Medicines do not save a life either promote or prevent epidemic diseases. Prescription should be right to bring the optimal benefit and should be safe, efficacious, cost-effective, and rational [2].

The World Health Organization (WHO) defined the rational use of the drug as patients receiving medications appropriate to their clinical needs in doses that meet their own individual requirements, for an adequate period and at the lowest cost to them and their community. Thus, drug utilization is an essential part to make patients beneficial by economically $[3,4]$. In general, the use of the least number of drugs to obtain the best possible effect in the shortest period and at a reasonable cost is called rational drug prescribing. Steps that are to be followed to fulfill the rational use of drugs are defining the patient's problems (diagnosis), defining effective and safe treatment (pharmacological and non-pharmacological), appropriate drugs, dosage, and duration, writing a good prescription to give patients adequate information, and planning to evaluate treatment responses $[5,6]$.

Irrational prescribing practices leads to ineffective and unsafe treatment, exacerbation or prolongation of illness, distress, and harm to the patient at a higher cost. Worldwide more than $50 \%$ of all medicines are prescribed, dispensed, or sold inappropriately, while $50 \%$ of patients fail to take them correctly [7]. These are common types of irrational medicine usage such as poly-pharmacy, inappropriate use of antimicrobials, inadequate dosage, overuse of injections, failure to prescribe in accordance with clinical guidelines, and self-medication. Irrational use of medicine may result in serious morbidity and mortality, additional economic, wastage of resources, increased treatment cost, increased risk for adverse drug reactions (ADRs), and emergence of drug resistance leads to a reduction in quality of drug therapy [8].

Psychiatric disorders are the major problem in this current scenario. Hence, the psychotropic agents were prescribed based on the different psychiatric disorders as depression, schizophrenia, anxiety, insomnia, panic attack, attention deficit hyperactivity disorder (ADHD), obsessivecompulsive disorder (OCD), etc. According to the results of World Mental Health Survey, psychiatric diseases are prevalent all around the world, and among them, mood disorders and anxiety disorder are the most common ones with, respectively, $16 \%$ and $12 \%$ lifetime prevalence rates. These disorders have several physical and social complications for the individuals' health state [9].

Numbers of drugs are available for treatment of depression and psychosis with or without complications. Prescription of such drugs varies from physician to physician depending on the health status and complications due to these diseases. It is essential to understand the trend of the prescribing guidelines indicators to help injudicious and rational use of drugs. Hence, this study was aimed to observe the prescribing pattern of psychotropic agents at the psychiatric department.

\section{METHODS}

This was a prospective and observational study, conducted in Psychiatric Department at Adichunchanagiri Institute of Medical Sciences, 
B.G Nagara, Mandya (D), Nagamangala (T), Karnataka, India, over a period of 6 months. It is a 1050 bedded tertiary care teaching hospital situated in a rural area having both outpatient and inpatient service. The study was initiated after obtaining approval from Institutional Ethical Committee of Adichunchanagiri Hospital and Research Hospital (AH and RC), B.G. Nagara.

\section{Study criteria}

\section{Inclusion criteria}

Both sexes and different age group patients attending the psychiatry department of the hospital who were prescribed medications consented to participate in the study.

\section{Exclusion criteria}

Patients who were pregnant, lactating, and unable to comply due to mental retardation, unconsciousness and who did not consent were not included in the study.

\section{Study procedure}

During the study period, all patients attending the psychiatric department who met the inclusion and exclusion criteria were enrolled into the study after obtaining the written consent. Patient's proforma such as demographic details, clinical data, diagnosis, medications used and side effects if any were collected by reviewing the patient's case notes/prescriptions had documented. Psychotropic medicine received by the study population in this study was ascertained by not only the type of disease, class of drugs, and frequency of the drugs and strength of the drugs in the prescribed pro forma. Thereby, the pattern of psychotropic agents used in different subsets of the population was analyzed.

Possible ADRs were assessed using various articles, standard textbooks like Martindale the complete drug reference 36 edition. The identified drug-related problems were discussed with the physicians for further management.

\section{Statistical methods}

The data were entered into Microsoft Excel 2010 and analyzed. The prescriptions were classified based on patient's age, gender, education background, religion, and types of disease. Microsoft word and excel have been used to construct bar graph and tables.

\section{RESULTS}

The study was a prospective and observational study. A total of 300 patients with a diagnosis of psychiatric disorders and who were receiving at least one Psychotropic drug were included in the study. The various details of the patients are showed in Table 1 . Table 1 summarized that out of 300 patients involved in the studies, $56.67 \%$ were male followed by female patients $43.33 \%$. The ratio of male to female participants was found to be 1.30:1. In the present study, the majority of the psychiatric patients were age group of 31-40 years (28.67\%) followed by $21-30$ years $(27.33 \%)$. The mean age of the participants was $38.14 \pm 8.43$ years. Majority of the participants were married 252 (84\%) and unemployed 156 (52\%). Only 1\% of the participants were employed. Majority of participants in the study were illiterate $225(75 \%)$ followed by literate $75(25 \%)$. The results were expressed as \pm standard error of the mean.

Out of 300 psychotropic patients, only a few patients 35 (11.67\%) had a family history of the psychiatric disorders, and majority of patients had no history of psychiatric disorders 165 (88.33\%). Among the psychotropic disease, the majority of patients were suffered from mood disorder 109 (36.33\%), a substance used and addictive disorder 52 (17.67\%) followed by Schizophrenia spectrum 46 (15.33\%). Results of clinical details of the study population are depicted in Table 2.

Out of 696 drugs prescribed, antidepressant 199 (28.59\%), benzodiazepines 117 (16.81\%), anticholinergic, and anticonvulsant $50(7.18 \%)$ each. Selective serotonin reuptake inhibitors (SSRIs) $(n=91)$ were highly prescribed antidepressant followed by tricyclic
Table 1: Demographic details of the patients

\begin{tabular}{ll}
\hline Characteristics & Frequency n (\%) \\
\hline Gender & \\
Male & $170(56.67)$ \\
Female & $130(43.33)$ \\
Total & $300(100.00)$ \\
Age group (years) & \\
10-20 & $28(9.33)$ \\
$21-30$ & $82(27.33)$ \\
$31-40$ & $86(28.67)$ \\
$41-50$ & $65(21.67)$ \\
$51-60$ & $25(8.33)$ \\
61-70 & $11(3.67)$ \\
$>70$ & $3(1.00)$ \\
Total & $300(100.00)$ \\
Marital status & \\
Married & $252(84.00)$ \\
Single & $48(16.00)$ \\
Total & $300(100.00)$ \\
Employment status & \\
Employed & $03(1.00)$ \\
Unemployed & $156(52.00)$ \\
Self-employed & $141(47.00)$ \\
Housewife & $37(12.33)$ \\
Business & $41(13.67)$ \\
Agriculture & $63(21.00)$ \\
Total & $300(100.00)$ \\
Education status & \\
Literate & $75(25.00)$ \\
Illiterate & $225(75.00)$ \\
Total & $300(100.00)$ \\
\hline &
\end{tabular}

Table 2: Clinical details of the patients

\begin{tabular}{ll}
\hline Characteristics & Frequency n (\%) \\
\hline Family history & \\
Yes & $35(11.67)$ \\
No & $265(88.33)$ \\
Psychiatric disorder & \\
Schizophrenia spectrum & $46(15.33)$ \\
Mood disorders & $109(36.33)$ \\
Anxiety disorder & $33(11.00)$ \\
OCD & $02(0.67)$ \\
Trauma and stress-related disorder & $05(1.67)$ \\
Seizures/personality disorder & $11(3.67)$ \\
Psychosomatic medicine & $22(7.33)$ \\
Disruptive impulse - control and conduct & $13(4.33)$ \\
disorder & \\
Substance used and additive disorder & $53(17.67)$ \\
Child psychiatry & $06(02)$ \\
Total & $300(100.00)$ \\
\hline
\end{tabular}

OCD: Obsessive-compulsive disorders

antidepressants drugs (TCAs) ( $\mathrm{n}=58)$. Other than psychotropic agents, $130(18.67 \%)$ patients received vitamin supplements followed by other drugs (proton pump inhibitor, nonsteroidal anti-inflammatory drugs) 25 (3.59\%). Table 3 summarized the prescription pattern of the different class of the agent used.

Out of 300 prescriptions, the total number of drugs prescribed was 696. Among them, $532(76.43 \%)$ drugs were psychotropic agents. Amitriptyline $31(53.44 \%)$ was highly prescribed TCAs class of antidepressant compared to opipramol 27 (46.55\%). Among all class of antidepressants prescribed escitalopram 59 (64.83\%) (SSRIs) was major class followed by mirtazapine $33(66 \%)$. Majority of typical antipsychotics prescribed were flupenthixol 11 (55\%) followed by haloperidol 8 (40\%) and chlorpromazine 1 (5\%).

Out of 82 atypical antipsychotics prescribed olanzapine was most frequently prescribed $47(57.31 \%)$ followed by risperidone 
22 (26.82\%). Lithium 11 (100) was only prescribed mood stabilizer. Among the anxiolytics as chlordiazepoxide 27 (90\%) was highly prescribed followed by alprazolam $3(10 \%)$. Out of 50 anticonvulsants drug, sodium valproate occupied highest rank 27 (54\%) followed by topiramate $8(16 \%)$ and phenytoin 8 (16\%) each. In Barbiturates class only phenobarbitone $3(100 \%)$ was prescribed. The anticholinergic drug, trihexyphenidyl $50(100 \%)$ was prescribed among 300 patients. Table 4 summarized commonly prescribed psychotropic agents in the study.

Table 5 summarized the total 214 antidepressant drugs were used in depression and bipolar affective disorder (BPAD). Among all drugs prescribed, escitalopram (SSRIs) was prescribed high number 38 (17.75) of a patient followed by Trihexyphenidyl (anticholinergic) 24 (11.68), olanzapine (atypical antipsychotic) 22 (10.28). Valproic acid $1(0.46 \%)$ was least prescribed agent for depression and BPAD.

Total number of 86 drugs were prescribed in Schizophrenia and psychosis followed by 1 drug prescribed in delusional disorder. Among the drug prescribed in schizophrenia and psychosis, trihexyphenidyl (anticholinergic) was highly prescribed 19 (22.09\%) followed by atypical antipsychotic drugs olanzapine $17(19.76 \%)$, risperidone $16(18.60 \%)$, and atypical antipsychotic drug flupenthixol 6 (6.97\%). In case of delusion disorder, only olanzapine $1(100 \%)$ was prescribed. Table 6 summarized the list of drugs which were prescribed in the subtype of psychiatric disorders.

In this study, we found 16 cases of panic disorder, where clonazepam was highly prescribed $13(81.25 \%)$ followed by amitriptyline $3(18.75 \%)$, 2 cases of agoraphobia (clonazepam and Mirtazapine were prescribed 1 each), 16 cases generalized anxiety disorder, where opipramol $9(56.25 \%)$ was highly prescribed followed by amitriptyline 4 (25\%) and sertraline $3(18.75 \%)$. In case of other anxiety disorder, the majority of SSRIs class drug escitalopram 5 (41.67\%) was highly prescribed followed by amitriptyline $3(25 \%)$ and sertraline and clonazepam $2(16.67 \%)$ each. Table 7 summarized the list of drugs prescribed in the subtype of anxiety disorder. Table 8 summarized only SSRIs class of drug sertraline $2(100 \%)$ was prescribed for OCD and related disorder.

Table 9 summarized that only 5 number of adjustment disorder were found in this which benzodiazepine class drug clonazepam was highly prescribed drug 2 (40\%) followed by TCAs drugs amitriptyline and opipramol 1 (20\%) each and atypical antipsychotic drug risperidone 1 (20\%).

Table 10 summarized the percentage of drugs prescribed in the subtype of seizures/personality/dissociative disorder. Out of 25 cases, 14 patients were having seizures disorder, and 11 patients were having dissociative/personality disorder. Anticonvulsant drugs carbamazepine and phenytoin $6(42.85 \%)$ each were highly prescribed followed by sodium valproate and clonazepam 1 (7.14\%) each. In dissociative/ personality disorder, atypical antipsychotic drug olanzapine 4 (46.36\%) was highly prescribed, and atypical antidepressant drug mirtazapine $1(9.09 \%)$ was least prescribed drug.

Table 11 summarized the list of drugs used in the treatment of psychosomatic disorders. Out of 34 psychosomatic disorders, 10 cases were somatic symptoms disorders, and 24 cases were pain disorder. In case of somatic symptoms disorders, amitriptyline 7 (70\%) was most frequently prescribed drug followed by opipramol $2(20 \%)$ and escitalopram $1(10 \%)$. In case of pain disorder, 6 different drugs were prescribed, out of which TCAs class drug amitriptyline 10 (41.67\%) was most frequently used followed by opipramol 5 (20.83\%).

A total number of 16 drugs was prescribed in impulsive suicidal attempt case, out of which (SSRIs) escitalopram 4 (25\%) and (atypical antidepressant) mirtazapine $4(25 \%)$ were most frequently prescribed drugs followed by topiramate 3 (18.75\%). Table 12 summarized drugs prescribed in the subtype of disruptive, impulse-control and conduct disorder.
Table 3: Prescription pattern of different class of drugs in the study

\begin{tabular}{ll}
\hline Drug class & Frequency n (\%) \\
\hline Antidepressants & $199(28.59)$ \\
TCAs & 58 \\
SSRIs & 91 \\
SNRIs & 17 \\
Atypical antidepressant & 33 \\
Antipsychotics & $102(14.65)$ \\
Typical & 20 \\
Atypical & 82 \\
Mood stabilizers & $11(1.58)$ \\
Anticholinergic & $50(7.18)$ \\
Benzodiazepines & $117(16.81)$ \\
Hypnotics & 41 \\
Anxiolytic & 30 \\
Anticonvulsant & 41 \\
Non-benzodiazepines & 05 \\
Anticonvulsants & $50(7.18)$ \\
Barbiturates & $03(0.43)$ \\
Vitamin supplements & $130(18.67)$ \\
Nicotinic antagonist & $09(1.29)$ \\
Others & $25(3.59)$ \\
Total & $696(100.00)$ \\
\hline
\end{tabular}

TCAs: Tricyclic antidepressants drugs, SSRIs: Selective serotonin reuptake inhibitors, SNRIs: Serotonin-norepinephrine reuptake inhibitors

Table 4: Commonly used psychotropic agents in the study

\begin{tabular}{lll}
\hline $\begin{array}{l}\text { Class of psychotropic } \\
\text { drug }\end{array}$ & Drug name & $\begin{array}{l}\text { Number of drugs } \\
\text { prescribed } \mathbf{n}(\%)\end{array}$ \\
\hline Antidepressants & Amitriptiline & $31(53.44)$ \\
TCAs & Opipramol & $27(46.55)$ \\
& Escitalopram & $59(64.83)$ \\
SSRIs & Sertraline & $32(35.16)$ \\
& Atomoxetine & $17(100)$ \\
SNRIs & Mirtazapine & $33(100)$ \\
Atypical & & \\
antidepressant & Haloperidol & $08(40)$ \\
Antipsychotics & Chlorpromazine & $01(5)$ \\
Typical & Flupenthixol & $11(55)$ \\
& Olanzapine & $47(57.31)$ \\
Atypical & Risperidone & $22(26.82)$ \\
& Quetiapine & $09(10.97)$ \\
& Aripiprazole & $02(2.43)$ \\
Mood stabilizer & Amisulpride & $02(2.43)$ \\
Benzodiazepine & Lithium & $11(100)$ \\
Anxiolytics & Chlordiazepoxide & $27(90)$ \\
& Alprazolam & $03(10)$ \\
Hypnotics & Lorazepam & $41(100)$ \\
Anticonvulsants & Clonazepam & $41(100)$ \\
Non-benzodiazepine & Zolpidem & $05(100)$ \\
Anticonvulsants & Sodium valproate & $27(54)$ \\
& Topiramate & $08(16)$ \\
& Valporic acid & $01(2)$ \\
& Carbamazepine & $06(12)$ \\
Barbiturates & Phenytoin & $08(16)$ \\
Anticholinergic & Phenobarbitone & $03(100)$ \\
& Trihexyphenidyl & $50(100)$ \\
\hline
\end{tabular}

TCAs: Tricyclic antidepressants drugs, SSRIs: Selective serotonin reuptake inhibitors, SNRIs: Serotonin-norepinephrine reuptake inhibitors

In case of alcohol dependence syndrome (ADS), the majority of benzodiazepine drug lorazepam $26(65 \%)$ was prescribed followed by chlordiazepoxide 13 (32.5\%). In case of ADS and tobacco dependence syndrome (TDS), anticonvulsant drug topiramate 5 (45.45\%) was 
Table 5: Antidepressant used (\%) in subtype of depressive disorder

\begin{tabular}{|c|c|c|c|}
\hline \multirow[t]{2}{*}{ Subtype of depressive disorder } & \multicolumn{2}{|l|}{ Medication used } & \multirow[t]{2}{*}{ Class of medication } \\
\hline & Drug (n=214) & n (\%) & \\
\hline \multirow{15}{*}{ Depression and BPAD } & Amitriptyline & $6(2.80)$ & TCAs \\
\hline & Escitalopram & $38(17.75)$ & SSRIs \\
\hline & Sertraline & $17(7.94)$ & SSRIs \\
\hline & Atomoxetine & $4(1.86)$ & SNRIs \\
\hline & Mirtazapine & $14(6.54)$ & Atypical antidepressant \\
\hline & Haloperidol & $4(1.86)$ & Typical antipsychotic \\
\hline & Flupenthixol & $5(2.33)$ & Typical antipsychotic \\
\hline & Olanzapine & $22(10.28)$ & Atypical antipsychotic \\
\hline & Risperidone & $4(1.86)$ & Atypical antipsychotic \\
\hline & Quetiapine & $6(2.80)$ & Atypical antipsychotic \\
\hline & Lithium & $11(5.14)$ & Mood stabilizers \\
\hline & Clonazepam & $17(7.94)$ & Benzodiazepine \\
\hline & Lorazepam & $10(4.67)$ & Benzodiazepine \\
\hline & Sodium valproate & $18(8.41)$ & Anticonvulsant \\
\hline & Trihexyphenidyl & $25(11.68)$ & Anticholinergic \\
\hline Total & & $214(100.00)$ & \\
\hline
\end{tabular}

BAPD: Bipolar affective disorder, TCAs: Tricyclic antidepressants drugs, SSRIs: Selective serotonin reuptake inhibitors, SNRIs: Serotonin-norepinephrine reuptake inhibitors

Table 6: Drugs used (\%) in subtype of psychiatric disorder

\begin{tabular}{|c|c|c|c|}
\hline \multirow[t]{2}{*}{ Subtype of psychosis disorder } & \multicolumn{2}{|l|}{ Medication used } & \multirow[t]{2}{*}{ Class of medication } \\
\hline & Drug & n (\%) & \\
\hline \multirow{13}{*}{$\begin{array}{l}\text { Schizophrenia and } \\
\text { psychosis }(n=86)\end{array}$} & Olanzapine & $17(19.76)$ & Atypical antipsychotic \\
\hline & Risperidone & $16(18.60)$ & Atypical antipsychotic \\
\hline & Quetiapine & $3(3.48)$ & Atypical antipsychotic \\
\hline & Flupenthixol & $6(6.97)$ & Typical antipsychotic \\
\hline & Haloperidol & $3(3.48)$ & Typical antipsychotic \\
\hline & Trihexyphenidyl & $19(22.09)$ & Anticholinergic \\
\hline & Lorazepam & $5(5.81)$ & Benzodiazepine \\
\hline & Clonazepam & $2(2.32)$ & Benzodiazepine \\
\hline & Escitalopram & $4(4.65)$ & SSRIs \\
\hline & Sertraline & $6(6.97)$ & SSRIs \\
\hline & Atomoxetine & $1(1.16)$ & SNRIs \\
\hline & Sodium valproate & $3(3.48)$ & Anticonvulsant \\
\hline & Aripiprazole & $1(1.16)$ & Atypical antipsychotic \\
\hline Total & & $86(100)$ & \\
\hline Delusional disorder & Olanzapine & $1(100)$ & Atypical antipsychotic \\
\hline
\end{tabular}

SSRIs: Selective serotonin reuptake inhibitors, SNRIs: Serotonin-norepinephrine reuptake inhibitors

Table 7: Drugs used (\%) in subtype of anxiety disorder

\begin{tabular}{llll}
\hline Subtype of anxiety disorder & Medication used & Class of medication \\
\cline { 2 - 3 } & Drug & $\mathbf{n ~ ( \% )}$ & \\
\hline Panic disorder $(\mathrm{n}=16)$ & Clonazepam & $13(81.25)$ & Benzodiazepine \\
Agorophobia $(\mathrm{n}=2)$ & Amitriptiline & $3(18.75)$ & TCAs \\
& Clonazepam & $1(50.0)$ & Benzodiazepine \\
GAD $(\mathrm{n}=16)$ & Mirtazapine & $1(50.0)$ & Atypical antidepressant \\
& Amitriptiline & $4(25.0)$ & TCAs \\
Other anxiety disorder $(\mathrm{n}=12)$ & Opipramol & $9(56.25)$ & TCAs \\
& Sertraline & $3(18.75)$ & SSRIs \\
& Escitalopram & $5(41.67)$ & SSRIs \\
& Sertraline & $2(16.67)$ & SSRIs \\
& Amitriptiline & $3(25.00)$ & TCAs \\
& Clonazepam & $2(16.67)$ & Benzodiazepine \\
\hline
\end{tabular}

GAD: Generalized anxiety disorder, TCAs: Tricyclic antidepressants drugs, SSRIs: Selective serotonin reuptake inhibitors

highly prescribed followed by serotonin-norepinephrine reuptake inhibitors (SNRIs) drug atomoxetine 4 (36.36\%). Out of 30 cases, TDS and nicotinic dependence syndrome, benzodiazepine drug lorazepam $15(50 \%)$ were most frequently prescribed followed by chlordiazepoxide 14 (46.67\%). Table 13 summarized drugs prescribed in the subtype of substance used and addictive disorder. 
Table 14 summarized drugs prescribed in the subtype of child psychiatry disorder. In this study, 4 cases of ADHD were found, and only atomoxetine (SNRIs) was prescribed.

Table 15 summarized that 300 prescriptions were analyzed in this study and total of 696 drugs were prescribed, i.e. 2.32 drugs per prescription. Out of 696 prescribed drugs, only 532 drugs were psychotropic drugs (64.28\%), where 498 drugs were administered orally and remaining 34 drugs were administered parenterally. Only $16.91 \%$ of psychotropic

Table 8: Drugs used (\%) in subtype of OCD and related disorder

\begin{tabular}{llll}
\hline Subtype of OCD & \multicolumn{2}{l}{ Medication used } & Class of medication \\
\cline { 2 - 3 } & Drug & $\mathbf{n}(\mathbf{\%})$ & \\
\hline OCD $(\mathrm{n}=2)$ & Sertraline & $2(100)$ & SSRIs \\
\hline
\end{tabular}

OCD: Obsessive-compulsive disorder, SSRIs: Selective serotonin reuptake inhibitors

Table 9: Drugs used (\%) in subtype of Trauma and stress-related disorder

\begin{tabular}{|c|c|c|c|}
\hline \multirow{2}{*}{$\begin{array}{l}\text { Subtype of trauma } \\
\text { and stress-related } \\
\text { disorder }\end{array}$} & \multicolumn{2}{|c|}{ Medication used } & \multirow{2}{*}{$\begin{array}{l}\text { Class of } \\
\text { medication }\end{array}$} \\
\hline & Drug & n (\%) & \\
\hline \multirow{5}{*}{$\begin{array}{l}\text { Adjustment } \\
\text { disorder }(n=5)\end{array}$} & Clonazepam & $2(40.0)$ & Benzodiazepine \\
\hline & Amitriptiline & $1(20.0)$ & TCAs \\
\hline & Opipramol & $1(20.0)$ & TCAs \\
\hline & Risperidone & $1(20.0)$ & $\begin{array}{l}\text { Atypical } \\
\text { antipsychotic }\end{array}$ \\
\hline & Total & 5 & \\
\hline
\end{tabular}

TCAs: Tricyclic antidepressants drugs drugs were prescribed in generic name and $64.28 \%$ these drugs were listed in essential drug list.

Out of 300 prescriptions (Table 16), 24.33\% of prescription contains only one drug, and $37 \%, 24 \%, 11.67 \%$, and $3 \%$ of prescriptions contains $2,3,4$, and 5 drugs, respectively. In this study, prescriptions containing 5 or more than 5 drugs were considered as polypharmacy 9 (3\%).

Table 17 summarized prescribing patterns based on the WHO core drug indicators. Out of 300 prescriptions, only $30 \%$ of prescriptions contain $<$ Rs. 100 and $70 \%$ of prescriptions contain more than 100 Rs. In this study, $94.33 \%$ prescription contains prescription errors.

\section{DISCUSSION}

The focus of our study was prescriptions given to consecutive patients consulting the psychiatric department for the $1^{\text {st }}$ time, irrespective of their past treatment history.

In this study, male participants were 170 (56.67\%), and female patients were 130 (43.33\%), indicating 6.67\% higher prevalence of psychiatric disorders in male population compared to the female population. Many studies have reported similar findings. This finding is similar to the study conducted by Mudhaliar et al. reported majority of participants were male (60.67\%) followed by female (39.33\%) [10]. And also supported by Grover et al. study where male participants were higher (54.8\%) compared to female participants (45.2\%) [11]. Hence, we concluded the male patient was more prescribed Psychotropic agents then female patients. In contrast to the present study, Thakkar et al. reported more female patients (56.67\%) visited the psychiatry OPD than men (43.33\%) [12]. From our study it was also found that prevalence of schizophrenia, anxiety disorder, seizures disorder, trauma/stressrelated disorder, substance used and addictive disorder and child psychiatry was higher in male patients compared to female, findings of which are similar to studies performed by Swamy et al. [13]. In contrast to our results, Thakkar et al. reported Mood disorder, Anxiety disorder and other psychotropic were high in female compared to male [12].

Table 10: Drugs used (\%) in subtype of seizures/personality/dissociative disorder

\begin{tabular}{|c|c|c|c|}
\hline \multirow[t]{2}{*}{ Subtype of seizures/personality/dissociative } & \multicolumn{2}{|l|}{ Medication used } & \multirow[t]{2}{*}{ Class of medication } \\
\hline & Drug & n (\%) & \\
\hline \multirow[t]{3}{*}{ Seizures disorder $(n=14)$} & Carbamazepine & $6(42.85)$ & Anticonvulsant \\
\hline & Sodium valproate & $1(7.14)$ & Anticonvulsant \\
\hline & Clonazepam & $1(7.14)$ & Benzodiazepine \\
\hline \multirow[t]{5}{*}{ Dissociative/personality disorder $(\mathrm{n}=11)$} & Olanzapine & $4(46.36)$ & Atypical antipsychotic \\
\hline & Trihexyphenidyl & $3(27.27)$ & Anticholinergic \\
\hline & Escitalopram & $3(27.27)$ & SSRIs \\
\hline & Mirtazapine & $1(9.09)$ & Atypical antidepressant \\
\hline & Total & 25 & \\
\hline
\end{tabular}

SSRIs: Selective serotonin reuptake inhibitors

Table 11: Drugs used (\%) in subtype of psychosomatic medicine

\begin{tabular}{|c|c|c|c|}
\hline \multirow{2}{*}{$\begin{array}{l}\text { Subtype of psychosomatic } \\
\text { medicine }\end{array}$} & \multicolumn{2}{|l|}{ Medication used } & \multirow[t]{2}{*}{ Class of medication } \\
\hline & Drug & n (\%) & \\
\hline \multirow[t]{2}{*}{ Somatic symptoms disorders $(n=10)$} & Opipramol & $2(20.00)$ & TCAs \\
\hline & Escitalopram & $1(10.00)$ & SSRIs \\
\hline \multirow{7}{*}{ Pain disorder $(n=24)$} & Amitriptyline & $10(41.67)$ & TCAs \\
\hline & Opipramol & $5(20.83)$ & TCAs \\
\hline & Sertraline & $2(8.33)$ & SSRIs \\
\hline & Clonazepam & $2(8.33)$ & Benzodiazepam \\
\hline & Mirtazapine & $2(8.33)$ & Atypical antidepressant \\
\hline & Sodium valproate & $3(12.5)$ & Anti-convulsant \\
\hline & Total & 34 & \\
\hline
\end{tabular}

TCAs: Tricyclic antidepressants drugs, SSRIs: Selective serotonin reuptake inhibitors 
Table 12: Drugs used (\%) in subtype of disruptive, impulse-control and conduct disorder

\begin{tabular}{|c|c|c|c|}
\hline \multirow{2}{*}{$\begin{array}{l}\text { Subtype of disruptive, impulse-control and conduct } \\
\text { disorder }\end{array}$} & \multicolumn{2}{|c|}{ Medication used } & \multirow[t]{2}{*}{ Class of medication } \\
\hline & Drug & n (\%) & \\
\hline \multirow{7}{*}{ Impulsive suicidal attempt $(\mathrm{n}=16)$} & Olanzapine & $1(6.25$ & Atypical antipsychotic \\
\hline & Lorazepam & $2(12.5)$ & Benzodiazepine \\
\hline & Escitalopram & $4(25.00)$ & SSRIs \\
\hline & Mirtazapine & $4(25.00)$ & Atypical antidepressant \\
\hline & Atomoxetine & $1(6.25)$ & SNRIs \\
\hline & Topiramate & $3(18.75)$ & Anti-convulsant \\
\hline & Total & 16 & \\
\hline
\end{tabular}

SSRIs: Selective serotonin reuptake inhibitors, SNRIs: Serotonin-norepinephrine reuptake inhibitors

Table 13: Drugs used (\%) in subtype of substance used and additive disorder

\begin{tabular}{|c|c|c|c|}
\hline \multirow[t]{2}{*}{ Subtype of substance used and addictive disorder } & \multicolumn{2}{|l|}{ Medication used } & \multirow[t]{2}{*}{ Class of medication } \\
\hline & Drug & n (\%) & \\
\hline \multirow[t]{3}{*}{$\operatorname{ADS}(n=40)$} & Chlordiazepoxide & $13(32.5)$ & Benzodiazepine \\
\hline & Lorazepam & $26(65.0)$ & Benzodiazepine \\
\hline & Haloperidol & $1(2.5)$ & Typical antipsychotic \\
\hline \multirow[t]{3}{*}{ ADS and TDS $(n=11)$} & Topiramate & $5(45.45)$ & Anticonvulsant \\
\hline & Atomoxetine & $4(36.36)$ & SNRIs \\
\hline & Olanzapine & $2(18.18)$ & Atypical antipsychotic \\
\hline \multirow{4}{*}{ TDS and NDS $(n=30)$} & Mirtazapine & $1(3.33)$ & Atypical antidepressant \\
\hline & Chlordiazepoxide & $14(46.67)$ & Benzodiazepine \\
\hline & Lorazepam & $15(50.0)$ & Benzodiazepine \\
\hline & Total & 81 & \\
\hline
\end{tabular}

ADS: Alcohol dependence syndrome, TDS: Tobacco dependence syndrome, NDS: Nicotinic dependence syndrome, SNRIs: Serotonin-norepinephrine reuptake inhibitors

Table 14: Drugs used (\%) in subtype of child psychiatry disorder

\begin{tabular}{llll}
\hline $\begin{array}{l}\text { Subtype of child } \\
\text { psychiatry disorder }\end{array}$ & \multicolumn{2}{l}{ Medication used } & $\begin{array}{l}\text { Class of } \\
\text { medication }\end{array}$ \\
\cline { 2 - 3 } & Drug & $\mathbf{n}(\%)$ & \\
\hline ADHD $(\mathrm{n}=4)$ & $\begin{array}{l}\text { Atomoxetine } \\
\text { Total }\end{array}$ & $4(100)$ & SNRIs \\
& 4 & \\
\hline
\end{tabular}

ADHD: Attention deficit hyperactivity disorder, SNRIs: Serotoninnorepinephrine reuptake inhibitors

Table 15: Analysis of prescribing indicators

\begin{tabular}{ll}
\hline Characteristics & Value \pm SEM \\
\hline Total number of prescriptions & 300 \\
Total number of drugs encountered & 696 \\
$\begin{array}{l}\text { Average number of drugs per prescription } \\
\text { Total number of psychotropic agents prescribed }\end{array}$ & $2.32 \pm 0.78$ \\
Total number of non-psychotropic agents & 164 \\
prescribed & \\
Percentage of psychotropic drugs prescribed by & 16.91 \\
generic name & \\
$\begin{array}{l}\text { Percentage of psychotropic drugs prescribed from } \\
\text { essential drug list }\end{array}$ & 64.28 \\
$\begin{array}{l}\text { Percentage of patients treated without drugs } \\
\text { Average consultant time for illness }\end{array}$ & 0.0 \\
$\begin{array}{l}\text { Number of orally administered drugs } \\
\text { Number of parenterally administered drug }\end{array}$ & $19 \pm 2$ min \\
\hline
\end{tabular}

SEM: Standard error of the mean

In our study, most of the age group of 31-40 years $(28.6 \%)$ were prescribed followed by age group 21-30 (27.33\%). The meant similar nature of finding was reported by Banerjee et al. that psychiatric disorder is commonly prevalent in patients below 40 years of age [14]. In another study, Grover et al. reported patients age in between 20 and 50 years $(89.4 \%)$ is most common in their study [11]. In our study schizophrenia, anxiety, seizures were most common in $41-50$ years patients.
Table 16: Number of drugs prescribed per prescription $(n=300)$

\begin{tabular}{lll}
\hline $\begin{array}{l}\text { Number of drugs } \\
\text { prescribed }\end{array}$ & $\begin{array}{l}\text { Number of } \\
\text { prescription (\%) }\end{array}$ & Polypharmacy ( $\mathbf{2 5})$ \\
\hline One & $73(24.33)$ & No \\
Two & $111(37.00)$ & No \\
Three & $72(24.00)$ & No \\
Four & $35(11.67)$ & No \\
Five & $09(3.00)$ & Yes \\
\hline
\end{tabular}

Table 17: WHO core drug use prescribing indicators

\begin{tabular}{|c|c|}
\hline Indicators & Values \pm SEM \\
\hline Average cost of medicine per day & Rs. $27.13 \pm 8.54$ \\
\hline Average cost of medicine per prescription & Rs. $286.55 \pm 20.39$ \\
\hline Percentage of prescriptions containing $<100$ & 30 \\
\hline Rs. & \\
\hline $\begin{array}{l}\text { Percentage of prescriptions containing more } \\
\text { than } 100 \text { Rs. }\end{array}$ & 70 \\
\hline $\begin{array}{l}\text { Percentage of prescription with } \\
\text { documentation error }\end{array}$ & 94.33 \\
\hline
\end{tabular}

SEM: Standard error of the mean, WHO: World Health Organization

Out of 300 participants, 252 (84\%) were married and remaining $48(16 \%)$ were unmarried. Most of the patients were married at a statistically significant rate in all type of disorder $(\mathrm{p}<0.0001)$, findings of these results are supported by Swamy et al. reported married (81.11\%) followed by single (14.85\%) and widow (2.9\%) [13]. In another study Grover et al. also reported married participants (71.8\%) were more common than unmarried single (22\%) [11].

Majority of patients were unemployed 156 (52\%) followed by selfemployed $141(47 \%)$ and employed $3(1 \%)$. Most of the patients were unemployed at a statistically significant rate in all type of disorder $(p=0.0475)$ except for the substance used and addictive disorder, unemployed participants were high in this study as the study was 
conducted in rural tertiary care hospital. Findings of our study are supported by Grover et al. study where 2289 (51.1\%) participants were unemployed followed by 2191 (48.9\%) participants were employed [11]. As far as employment status of the patient is concerned, except substance used and addictive disorder almost all psychiatric disorders are commonly prevalent in unemployed patients.

In our study illiterate 225 (75\%) followed by literate $(25 \%)$ due to study was conducted in a rural area where majority of participants were farmer, housewives, and non-proper schooling facility. Most of the patient were illiterate at a statistically significant rate $(\mathrm{p}=0.0060)$ in all type of disorder except for trauma/stress-related disorder where illiterate and literate patient were equal in number.

Out of 300 prescriptions, total numbers of 696 drugs were prescribed. The types and number of drugs prescribed in the study are antidepressants 199 (28.59\%), vitamin supplements 130 (18.67\%), benzodiazepines 117 (16.81\%), antipsychotics 102 (14.65\%), anticholinergic 50 (7.18\%), mood stabilizers 11 (1.58\%), Nicotinic antagonistic 9 (1.59\%), barbiturates $3(0.43 \%)$, and others drugs 25 (3.59\%). Vitamins supplements were prescribed commonly among ADS and patient getting psychotropic agent due to fulfill thiamine deficiency and inhibit gastric irritation, respectively. Some antidepressants or psychotropic agents may induce gastric irritations.

Out of 696 prescribed drugs, a total number of psychotropic agents prescribed were 532. Among 532 psychotropic agents, 498 (93.61\%) drugs were prescribed in tablet dosage form followed by injections 34 (6.39\%). Among antidepressants $(n=199)$, SSRIs class $(n=91)$ of antidepressants were most frequently prescribed followed by TCAs $(\mathrm{n}=58)$, atypical antidepressants $(\mathrm{n}=33)$ and SNRIs $(\mathrm{n}=17)$. This finding was supported by Mishra et al. were they reported $58.87 \%$ of SSRIs prescribed [15]. Amitriptyline 31 (53.44\%) was most commonly prescribed TCAs class antidepressants followed by opipramol 27 (46.55). Among SSRIs class antidepressants, escitalopram $59(64.83 \%)$ was highly prescribed followed by sertraline 32 (35.16\%). Flupenthixol $11(55 \%)$ was commonly prescribed typical antipsychotics followed by haloperidol $8(40 \%)$ and $1(5 \%)$. Among all atypical antipsychotics prescribed olanzapine $47(57.31 \%)$ was prescribed commonly followed by risperidone $22(26.82 \%)$. These results were supported by Roopadevi et al. study [16]. Lithium was the most common mood stabilizer drug being prescribed $11(100 \%)$. Lorazepam $(n=41)$ and clonazepam $(n=41)$ were most common benzodiazepines prescribed followed by chlordiazepoxide $(n=27)$, zolpidem $(n=5)$, and alprazolam $(n=3)$. Trihexyphenidyl was only anticholinergic drug which was prescribed.

Out of 300 prescriptions, $73(24.33 \%)$ prescriptions contain only one drug, $111(37.0 \%)$ contains 2 drugs, 72 (24\%) contains 3 drugs, $35(11.67 \%)$ contains 4 drugs, and $9(3 \%)$ contains 5 drugs. In this study, prescriptions containing 5 or more than 5 drugs were considered as polypharmacy.

The average number of drugs per prescription was lower $(2.32 \pm 0.78)$ than that found in the studies conducted by Mudhaliar et al., where it was reported 2.37 drugs per prescription [10]. In contrast to our results, Swamy et al. [13] and Ghosh and Roychaudhury [17] reported $1.85 \pm 0.8$ and 2.03 drugs per prescriptions. During the study, it was also observed that $156(52 \%)$ prescriptions contain drug combinations. In our setting very, less number of drugs (16.91\%) were prescribed by generic names, which is supported by the study conducted in Nigeria, Ghana, Lebanon, and Nepal. In contrast to this study, Mudhaliar et al. [10] and Mishra et al. [15] reported 99.08\% and 99\%, respectively, were prescribed in generic form. This study suggested that substitution of branded drug by generic drugs helps in decreasing the overall cost of the drug therapy and treatment cost and is hence recommended. However, there have been concerns in the case of narrow therapeutic index drugs. As per Thakkar et al., generic substitution can be beneficial, provided adequate quality control is assured [12]. Out of 532 Psychotropic drugs, $64.28 \%$ of drugs were prescribed from essential drug list (WHO; 2013), findings of these studies were similar to the study conducted by Mishra et al. (44.99\%) [15]. The primary purpose of National list of essential medicine is to promote rational use of medicines considering the three important aspects, i.e. cost, safety, and efficacy. Only pharmacotherapy and patient counselling was done during the study. Average psychiatric consultations time was $19 \pm 2 \mathrm{~min}$ where all patients got at least one drug. Almost all patients sought to get counselling advice about medication and treatment therapy with us. Based on the severity of cases some patients were given injections. During this study out of 532 psychotropic drugs used, 34 (6.39\%) of medication were given in injection form.

Some problem based complementary indicators were used to assess drug use in prescription and majority of the prescriptions were incomplete 283 (94.33\%), where most of the cases weight and route of administration were not mentioned 185 (61.67\%). Even though such errors seem to minor error but might cause harm during overall treatment of patients.

\section{CONCLUSION}

Drug utilization is an evolving process. Thus, continuous monitoring of prescription pattern helps to understand changing trends in prescribing pattern over time. Our study was mainly focused on the prescribing pattern of psychotropic agents at psychiatric department of rural tertiary care teaching hospital. Such study provides opportunities for enhancing the quality of mental health care in our society, by providing awareness in rational and cost-effective use of medicine.

\section{AUTHORS CONTRIBUTION}

Madan Rayamajhi, Shahinur Islam and Nanjunda N -Principal Investigator who conducted the study.

\section{CONFLICTS OF INTEREST}

The authors have no personal, professional, or ethical conflicts of interest in the publication of this study.

\section{REFERENCES}

1. Welcome MO. The Nigerian health care system: Need for integrating adequate medical intelligence and surveillance systems. J Pharm Bioallied Sci 2011;3:470-8.

2. Kar SS, Pradhan HS, Mohanta GP. The concept of essential medicines and rational use in public health. Indian J Community Med 2010;35:10-3.

3. Prakash B, Nadig P, Nayak A. Rational prescription for a dermatologist. Indian J Dermatol 2016;61:32-8.

4. Ilyaz M, Baig MM, Ramakrishna R, Quadir MA, Fathima M, Khan SA. Drug utilization study of antipsychotics and its common ADR's in the psychiatry OPD of OHRC. Int J Pharm Pharm Sci 2014;6:162-5.

5. Paul PK, Konwar M, Das S. To study the prescribing pattern of antipsychotic drugs in a tertiary care hospital of Assam. Int J Pharm Pharm Sci 2014;6:435-7.

6. Nagaraja PS, Shreenivas P, Vedavathi H, Chidanand KN, Murray JL, Manjunath H. A study on prescription analysis and utilization of antibiotics in geriatric in-patients admitted in Shimoga institute of medical sciences tertiary care hospital, Shimoga, Karnataka, India. Int J Basic Clin Pharmacol 2016;5:904-9.

7. Babar HS, Hussain S, Maqsood Z, Dad HA, Khan M, Rahman AA, et al. Adherence to prescription format and compliance with who core prescribing indicators. Pharm Sci Res. 2014;6(4):195-9.

8. Gopalakrishnan S, Ganeshkumar P, Katta A. Assessment of prescribing practices among urban and rural general practitioners in Tamil Nadu. Indian J Pharmacol 2013;45:252-7.

9. Kessler RC, Aguilar-Gaxiola S, Alonso J, Chatterji S, Lee S, Ormel J, et al. The global burden of mental disorders: An update from the WHO world mental health (WMH) surveys. Epidemiol Psichiatr Soc 2009;18:23-33. 
10. Mudhaliar MR, Ghouse ISM, Sadubugga P, Narala SR, Chinnakotla V, Yendluri P. Psychotropic drug utilization in psychiatric outpatient department of a tertiary care teaching hospital in India. Int J Res Med Sci. 2017;5(4):1612-16.

11. Grover S, Kumar V, Avasthi A, Kulhara P. An audit of the first prescription of new patients attending a psychiatry walk-in-clinic in north India. Indian J Pharmacol 2012;44:319-25.

12. Thakkar BK, Mangal JM, Gauri B, Abhijit J, Khobragade A. A drug utilization study of psychotropic drugs prescribed in the psychiatry outpatient department of a tertiary care hospital. J Clin Diag Res 2013;7:2759-64.

13. Swamy MK, Wagle L, Giri V. Drug utilisation pattern of psychotropic drugs in psychiatric outpatient department of rural tertiary care teaching hospital. Int J Pharm Sci Res 2016;7:3497-8.
14. Banerjee I, Roy B, Sathian B, Banerjee I, Chakraborty PK, Saha A. Socio-demographic profile and utilization pattern of antipsychotic drugs among schizophrenic inpatients: A cross-sectional study from western region of Nepal. BMC Psychiatry 2013;13:1-10.

15. Mishra S, Swain T, Mohanty T. Patterns of prescription and efficacy evaluation of antidepressants in a tertiary care teaching hospital in Eastern India. Asian J Pharm Clin Res 2012;5:193-6.

16. Roopadevi HS, Ramesh KS, Nagabushan H. Pattern of psychotropic prescription in a tertiary care teaching hospital: A critical analysis. Asian J Pharm Clin Res 2015;8:252-55.

17. Ghosh S, Roychaudhury S. Prescribing pattern of antidepressant drugs in a tertiary care hospital of eastern India. J Chem Pharm Res 2014;6:2593-7. 